\title{
Do Silent Brain Infarctions Predict the Development of Dementia After First Ischemic Stroke?
}

\author{
Ivan Barbov ${ }^{1}$, Slavica Arsova $^{2}$, Arben Taravari $^{1}$, Vera Petrova $^{1}$ \\ ${ }^{1}$ University Clinic of Neurology, Cerebrovascular Disorders, Faculty of Medicine, Ss Cyril and Methodius University of \\ Skopje, Republic of Macedonia; ${ }^{2}$ University Clinic for Psychiatry, Faculty of Medicine, Ss Cyril and Methodius University of \\ Skopje, Republic of Macedonia
}

\begin{abstract}
Citation: Barbov I, Arsova S, Taravari A, Petrova V. Do Silent Brain Infarctions Predic the Development of Dementia After Firs Ischemic Stroke? OA Maced J Med Sci. 2013 Dec 15; 1(1):66-69. http://dx.doi.org/10.3889/oamjms.2013.013

Key words: silent infarcts; dementia; ischemic stroke; vascular risk factors; cognitive decline. "Correspondence: Dr. Ivan Barbov. Clinic of Neurology, Cerebrovascular Disorders, Vodnjanska 17, Skopje 1109, Republic of Macedonia. Phone: +38978444161. E-Mail dr_barbov@yahoo.com

Received: 03-Nov-2013; Revised: 18-Nov2013; Accepted: 19-Nov-2013; Online first: 27-Nov-2013

Copyright: ๑ 2013 Barbov I. This is an openaccess article distributed under the terms of the Creative Commons Attribution License, which permits unrestricted use, distribution, and reproduction in any medium, provided the original author and source are credited.

Competing Interests: The authors have declared that no competing interests exist.
\end{abstract}

\begin{abstract}
Background: Silent brain infarctions ( $\mathrm{SBI}$ ) are common findings in advanced age, but their relationship to dementia is still uncertain.

Aim: The present study was designed to evaluate whether SBI predict the development of dementia after first clinical ischemic stroke.

Methods: We blindly studied admission CT scans of 102 consecutive nondemented patients presenting with ischemic stroke that clinically was their first stroke episode. SBI were defined as CT evidence of infarcts not compatible with the acute event. The patients were subsequently followed for their mental state for 2 years. Survival analysis, wherein onset of dementia was the end point, was performed on the total sample population and conducted separately on those with and without $\mathrm{SBI}$ at admission.
\end{abstract}

Results: Dementia developed in 33 patients (32.3\%), including 17 of the $37(45.9 \%)$ with SBI and 16 of the $65(24.6 \%)$ without SBI. Thus, dementia was strong related to SBI.

Conclusion: Elderly people with silent brain infarcts and stroke have an increased risk of dementia and a steeper decline in cognitive function than those without such lesions.

\section{Introduction}

With advancements in neuroimaging, silent brain infarctions (SBI) were recognized as a common finding in the elderly, including patients with first-ever symptomatic stroke [1]. Data are available concerning prevalence, risk factors, and CT characteristics of SBI in patients with ischemic stroke, but the clinical significance of $\mathrm{SBI}$ is still controversial. It would not be surprising if such silent infarctions could predict the subsequent development of dementia. The possibility that $\mathrm{SBI}$ may indicate predisposition to dementia is important because cognitive impairment after ischemic stroke is frequent, but factors leading to its occurrence are poorly understood. Furthermore, several recent studies showed that dementia after stroke does not necessarily appear to have "typical" vascular features, such as acute onset. In the present study we assessed the predictive value of $\mathrm{SBI}$ in relation to the development of cognitive decline in patients after what clinically appeared to be their firstever ischemic stroke [2-5].

\section{Subjects and Methods}

This is a prospective study aimed at determining whether there is a relationship between $\mathrm{SBI}$ and any future development of dementia after clinical stroke.

Between January 2009 and December 2010 , 258 patients with acute cerebrovascular disease were admitted to our department. Of these, 36 (13.9\%) had 
transient ischemic attacks and $16(6.2 \%)$ had cerebral hemorrhage. Among the 206 patients with ischemic stroke, 16 (6.2\%) died during hospitalization, 51 $(19.8 \%)$ had severe aphasia or coexisting cerebral or serious medical disorders, 18 (7.0\%) reported previous cerebral insults, and 19 (7.4\%) were reported to have had cognitive impairment before the stroke. The present study included the remaining 102 patients with clinical first-ever ischemic stroke, underwent brain CT examinations within 48 hours of admission and who were followed up later.

The patients' mean \pm SD age was $72.3 \pm 6.8$ years. All were examined and evaluated according to a standard protocol, including medical and neurological examinations as well as a Mini Mental State Examination Test [6] (MMSE) including 30 questions regarding orientation, registration, attention, calculation, general knowledge, recent memory, language through naming, repetition, comprehension of verbal commands, writing and reading, constructional abilities, and abstraction. The patients were followed up with biannual evaluations of their mental status for 2 years. The diagnosis of dementia was based on criteria according to the Diagnostic and Statistical Manual of Mental Disorders, edition 4, revised [7]. CT examinations were performed shortly after admission. Contrast material was not used routinely. The type, size, and vascular territory of the strokes were recorded. SBI was diagnosed if the CT examination revealed an infarct in a site not compatible with the clinical symptoms. For the present analysis, all scans were retrieved and read by the same neuroradiologist, who had no knowledge of the clinical data [8].

\section{Statistical Analysis}

The Cox regression analysis was used to control for any differences between the groups in age, sex, and major vascular risk factors. The prevalence of SBI among the patients who did or did not develop dementia was compared by a nonparametric test $\left(x^{2}\right)$. Patients who died or were lost to follow-up without developing dementia during the 2-year period were censored at the time of their last examination.

\section{Results}

Dementia developed in 33 patients (32.3\%), including 17 of the 37 (45.9\%) with SBI and 16 of the $65(24.6 \%)$ without SBI (Table 1$)$. There were no differences in age or sex in the demented and nondemented patients (Table 2). Statistically significant differences were found in the two groups pertaining to major vascular risk factors (arterial hypertension, atrial fibrillation, diabetes mellitus, hyperlipidemia, ischemic heart disease).

As for the noncensored data of patients with SBI, 17 of 37 (46\%) subsequently developed dementia as opposed to 16 of 65 (25\%) without SBI. Obviously, SBI affect subsequent cognitive decline in these patients, Odds Ratio=11.60 Wald 95\%: Cl: 3.07 $<\mathrm{OR}<69.37$. It is statistically significant for $P<0.01$. Seventy-eight percent of the SBI were lacunar and $22 \%$ were cortical, with no differences in later development of dementia $(P>0.04)$. We found no relation between a decline in the score on the MiniMental State Examination and the presence of silent brain infarcts $(P>0.05)$.

Table 1: Silent Brain Infarctions and Subsequent Development of Dementia After First-Ever Ischemic Stroke.

\begin{tabular}{|c|c|c|c|c|}
\hline \multirow{2}{*}{ SBI } & \multicolumn{2}{|c|}{ Subsequent Dementia } & \multirow{2}{*}{$\begin{array}{c}\text { Censored }^{1} \\
(n=10)\end{array}$} & \multirow{2}{*}{$\begin{array}{c}\text { All Patients } \\
(n=102)\end{array}$} \\
\hline & No $(n=59)$ & Yes $(n=33)$ & & \\
\hline No & 43 & 16 & 6 & 65 \\
\hline Yes & 16 & 17 & 4 & 37 \\
\hline
\end{tabular}

$\mathrm{SBI}$, silent brain infarctions; ${ }^{1}$ those patients who died $(n=8)$ during the follow-up period before they developed dementia or were lost to follow-up $(n=2)$.

The number of SBI also predicts the development of dementia. Thus, in 21 patients with a single SBI, 8 (38\%) developed dementia compared with 16 patients who presented with two of more SBI, of whom $9(56 \%)$ developed dementia. It is statistically significant for $P<0.01$. Of the 65 patients with no SBI, $16(24 \%)$ developed dementia, without bigger statistical significance ( $p>0.05)$.

Table 2: Mean age and sex of patients with and without dementia after first-ever ischemic stroke.

\begin{tabular}{lccc}
\hline & \multicolumn{2}{c}{ Subsequent Dementia } & \multirow{2}{*}{ All Patients ${ }^{1}(\mathbf{n}=\mathbf{1 0 2})$} \\
\cline { 2 - 3 } & No $(\mathbf{n}=\mathbf{5 9})$ & Yes $(\mathbf{n}=\mathbf{3 3})$ & \\
\hline Men & $32(54 \%)$ & $18(54 \%)$ & 50 \\
Women & $27(46 \%)$ & $15(46 \%)$ & 42 \\
Mean \pm SD age, y & $71.2 \pm 8.5$ & $73.3 \pm 5.3$ & $72.3 \pm 6.8$ \\
\hline
\end{tabular}

${ }^{1}$,Includes only noncensored data.

\section{Discussion}

Previous studies have demonstrated the equivocal value of $\mathrm{SBI}$ in predicting the development of dementia after stroke. However, all recent reports are based on different groups of patients with a different incidence of dementia. Fukuda et al. [9] demonstrated a correlation between mental deterioration and severity of lesions in white matter. Their study was based on patients with symptomatic multiple lacunar infarcts rather than SBI. However, almost all authors indicated the necessity of establishing a relationship between SBI and dementia by evaluating an adequate number of patients with and without dementia and, in parallel, an adequate number of patients with and without SBI.

This is a prospective study aimed at determining whether there is a relationship between $\mathrm{SBI}$ and any future development of dementia after clinical stroke.

Our study included a sufficient number of patients in each previously delineated group and demonstrated a strong correlation between SBI and the development of dementia after first-ever 
symptomatic ischemic stroke $(P<0.01)$. Wolfe et al. noted that the effects of multiple infarcts are synergistic and not simply additive.

In a previous study we investigated the likelihood of subclinical cognitive dysfunction due to primary degenerative dementia in those patients who subsequently developed cognitive decline after stroke. The present results support this view and perhaps may be useful to define the causes of cognitive impairment after stroke. Stephen et al. [10] concluded that SBI influence the prognosis of stroke or neurological outcome, and the present study additionally shows that SBI are significant in the development of poststroke dementia.

People with silent infarcts had a steeper decline in cognitive function than those without silent infarcts, but this decline was confined to people who had additional silent brain infarcts after base line [11].

The dementia diagnoses in our study were clinical diagnoses. We intentionally refrained from analyzing subtypes of dementia, because a distinction based on clinical information is hard to make, especially in elderly people, in whom dementia often is a heterogeneous disorder [12]. There is increasing evidence that vascular factors may contribute to the development of Alzheimer's disease. After a stroke, dementia, including Alzheimer's disease, develops in approximately 30 percent of patients with symptomatic infarcts. We found that silent brain infarcts increase the risk of dementia [12].

Our findings of a large number of new infarcts in the participants in whom dementia developed and a steeper decline in cognition in those with a new infarct support the notion that people with silent brain infarcts are at high risk for additional infarcts, both silent and symptomatic, which may contribute to dementia. Perhaps an infarct in a brain already affected by Alzheimer's disease-related abnormalities further impairs cognition, leading to clinically evident dementia. This notion is supported by autopsy findings showing that fewer plaques and tangles led to clinical Alzheimer's disease in the presence of lacunar infarcts. Alternatively, silent brain infarcts may trigger the development of senile plaques and neurofibrillary tangles or reflect cerebral vulnerability or a certain vascular risk profile that enhances the abnormalities associated with Alzheimer's disease [13].

The reason that we found no relation between a decline in the score on the Mini-Mental State Examination and the presence of silent brain infarcts is probably that this test, although useful as a screening tool for dementia, is not a very sensitive means of detecting subtle changes in cognitive function.

A lot of studies showed a relationship between dementia occurrence and volume or strategic location of infarction, a host of cranial CT and MRI [14]. These studies provide evidence that dementia related to stroke is associated with higher mean tissue loss, infarct number, location (eg, dominant thalamus and angular gyrus, bilateral infarcts, deep frontal infarcts, left hemisphere infarcts), and degree of "cerebral atrophy" as demonstated by ventricular size, area of subarachnoid space, and size of the third ventricle.

In recent years, there has been evidence demonstrating that vascular risk factors (VRF) were associated with dementia after stroke. Hypertension, diabetes and hypercholesterolemia were found to be associated with the development of dementia. There are limited studies on the association between VRF and the progression of dementia. Recently recurrent stroke and atrial fibrillation were found to be associated with the progression from Mild Cognitive Impairment $(\mathrm{MCl})$ to dementia. However, it remains unknown whether untreated VRF hastens the conversion from $\mathrm{MCl}$ to dementia, and, in particular, whether the treatment of VRF can halt the development of incident post stroke dementia [15].

As a conclusion, elderly people with silent brain infarcts and stroke have an increased risk of dementia and a steeper decline in cognitive function than those without such lesions. The presence of silent brain infarcts on CT identifies persons at increased risk for dementia, probably because these people continue to have additional brain infarcts, both silent and symptomatic, that decrease their cognitive function.

\section{References}

1. Desmond DW, Moroney JT, Paik MC, et al. Frequency and clinical determinants of dementia after ischemic stroke. Neurology. 2000;54:1124-31.

2. Yannick B, Corine AE, Jerome D, et al. Prevalence of Early Dementia After First-Ever Stroke: A 24-Year Population-Based Study Stroke. 2011;42:607-612.

3. Sarah EV, Niels DP, Tom den $\mathrm{H}$, et al. Silent Brain Infarcts and the Risk of Dementia and Cognitive Decline. N Engl J Med. 2003; 348:1215-22.

4. Henon H, Durieu I, Guerouaou D, et al. Poststroke dementia: incidence and relationship to prestroke cognitive decline. Neurology. 2001;57:1216-22.

5. Caplan LR. Silent brain infarcts. Cerebrovasc Dis. 1994;4(Suppl 1):32-39.

6. Folstein M, Folstein S, McHugh PR. Mini-Mental State: a practical method for grading the cognitive state of patients for the clinician. J Psychiatr Res. 1975;12:189-192.

7. American Psychiatric Association. Diagnostic and Statistical Manual of Mental Disorders, Fourth Edition (DSM-IV). Washington, DC: American Psychiatric Association, 1994:143147.

8. Boon A, Lodder J, Heuts-van Raak L, Kessels F. Silent brain infarcts in 755 consecutive patients with a first-ever supratentorial ischemic stroke. Stroke. 1994:25:2384-90.

9. Fukuda H, Kobayashi S, Okada K, Tsunematsu T. Frontal white matter lesions and dementia in lacunar infarction. Stroke. 1990;21:1143-49.

10. Stephen DJM, Sarah T, Martin SD, et al. Cognitive impairment 
after lacunar stroke: systematic review and meta-analysis of incidence, prevalence and comparison with other stroke subtypes J Neurol Neurosurg Psychiatry. 2013;84:893-900.

11. de Groot JC, de Leeuw FE, Oudkerk M, et al. Periventricular cerebral white matter lesions predict rate of cognitive decline. Ann Neurol. 2002;52:335-41.

12. Neuropathology Group of the Medical Research Council Cognitive Function and Ageing Study (MRC CFAS). Pathological correlates of late-onset dementia in a multicentre, community-based population in England and Wales. Lancet. 2001;357:169-75

13. Vermeer SE, Koudstaal PJ, Oudkerk M, et al. Prevalence and risk factors of silent brain infarcts in the population-based Rotterdam Scan Study. Stroke. 2002;33:21-25.

14. Bernick $C$, Kuller L, Dulberg $C$, et al. Silent MRI infarcts and the risk of future stroke: the Cardiovascular Health Study. Neurology. 2001;57:1222-29.

15. Salka SS, Wiesje MF, Elisabeth CS, et al. Neurological Signs in Relation to Type of Cerebrovascular Disease in Vascular Dementia Stroke. 2008;39:317-322. 